\title{
Pandas: A Rare Case Report
}

\author{
Y. Kulkarni, R. Warkari \\ ${ }^{1}$ Associate Professor, Department of Psychiatry, Padmshree Dr. D. Y.Patil Hospital and Research Institute, D. Y. Patil University, Kolhapur \\ -416006 (India). \\ ${ }^{2}$ Junior Resident ( $3^{\text {rd }}$ year), Department of Psychiatry, Padmshree Dr. D. Y. Patil Hospital and Research Institute, D. Y. Patil University, \\ Kolhapur - 416006 (India)
}

\begin{abstract}
PANDAS,also labelled as PANS,is a pediatric autoimmune neuropsychiatric disorder due to streptococcal infection. Although it affects 1 in 1000 children, cases reported in Indian context are relatively few because of lack of awareness about the syndrome amongst clinicians. We report here a case of a 13 years old male from a rural area presenting to us with an acute onset of repetitive behavior following a febrile illness 2 months prior. Investigations confirmed rheumaticvalvular heart disease. Adiagnosis of PANDAS was made and treatment was started. The patient responded to treatment and gained symptomatic relief. Timely intervention and high index of suspicion towards a pediatric febrile illness can help to reduce the morbidity associated with this neuropsychiatric disorder.
\end{abstract}

Keywords: Acute onset, early adolescence,PANDAS, PANS, repetitive behavior, rheumatic heart disease

\section{Introduction}

PANDAS is an acronym for pediatric autoimmune neuropsychiatric disorder due to streptococcal infection. Also labelled as PANS i.e. pediatric acute onset neuropsychiatric syndrome [1] which applies to children and adolescents who suddenly develop obsessive compulsive symptoms or tics or abnormal eating behavior along with other symptoms which may include-

- Severe separation anxiety

- Trouble eating

- Bed wetting

- Deterioration in reading and writing abilities

- Irritability

- Panic attacks

- Suicidal thoughts.

It happens in about 1 in 1000 children and thrice more often in boys than girls. It is said to be a pre-pubertal condition, since most children have developed immunity to streptococcus by adolescence. Hence,the onset is not likely to occur thereafter, though PANDAS symptoms may continue to occur intermittently.

Some of the theories premise that our immune system recognizes bacteria and produces antibodies to fight them but because of the similarities to human cells those antibodies in turn sometimes "misrecognize" cells in a child's own body and attack them. In rheumatic heart disease, they attack the heart while in PANDAS it is considered to be the basal ganglia in brain [2].
Since the constellation of the symptoms can be debilitating at times, it is important to be aware of this disorder. In order to overcome lesser understanding of this topic and to affirm the existing database we report here a case that we recently came across.

\section{Case Report}

A $13 y r s$ old male student of $8^{\text {th }}$ standard, hailing from a rural area, presented to us with an acute onset of repetitive picking up of his clothes in order to rearrange them. Prevention in doing so resulted in irritability, apparently due to an urge which he was unable to control. There were frequent violent outbursts. On a few occasions, he even physically assaulted his mother. He was also noticed to remain immobile for long periods of time. There was significant social and scholastic deterioration with a fortnight of missed school. His sleep and appetite were significantly reduced.

Around 2 months back he developed sore throat, pain in both knees, shoulders and at metacarpophalangeal joints along with high grade fever. A pediatrician treated him as an indoor patient with antibiotics without investigating in detail. During treatment, his general condition improved and fever subsided. Immediately after the discharge from the pediatric nursing home, he developed the aforementioned symptoms for which a general practitioner referred him to our unit. There was no history of similar episodes in the past, seizures, urinary incontinence, head injury, any previous psychiatric illnessor intellectual disability. Developmental and family history were unremarkable. 


\section{International Journal of Science and Research (IJSR) \\ ISSN (Online): 2319-7064}

Index Copernicus Value (2013): 6.14 | Impact Factor (2014): 5.611

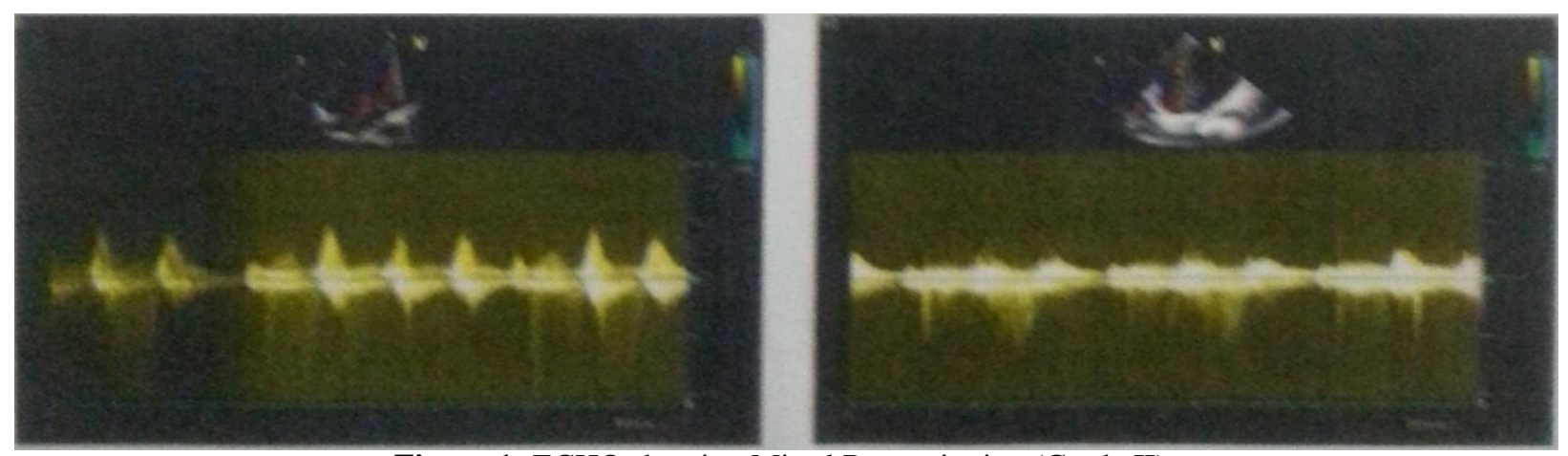

Figure 1: ECHO showing Mitral Regurgitation (Grade II)

Physical examination of the patient revealed pallor, swelling over both knees and on auscultation early systolic murmur was present. His vitals were within normal limits. Before initiating the treatment patient was investigated for ASO titer, 2-D echocardiography and a CAT scan of the Brain. The ASO titer was markedly raised up to $1600 \mathrm{IU} / \mathrm{ml}$. 2-D echo concluded mitral regurgitation of Grade 2 with intact left ventricular function. CT-Brain was unremarkable. A diagnosis of PANDAS was made and treatment was started. He was placed on Cap. Fluoxetine 20mg, Tab. Olanzepine $2.5 \mathrm{mg}$ and Inj. Benzathine penicillin G 1.2 million units once every 3 weeks as per consultation with the Cardiologist. Patient responded well to treatment. His repetitive behavior and irritability reduced significantly over a period of 7-10days.

\section{Discussion}

So far, only a few cases of PANDAS have been reported and none of these cases could classically fit into the specific diagnostic criteria for PANDAS except a case reported in Journal of Clinical and Diagnostic Research by Mona Srivastava et al. in 2011[3]. Recently BaljeetMaini also reported a case of pediatric autoimmune neuropsychiatric disorder after streptococcal infection in which the onset of tics was within a week of a febrile illness [4].The case which was described by Shankarnarayan and John had an adult onset of obsessive compulsive features instead of tics [5]. On the contrary, in our case though the repetitive behavior of the child was not fully satisfying the criteria for compulsion; scholastic, interpersonal and neurocognitive functions were significantly impaired. The symptoms were acute in onset and were preceded by the streptococcal infection which can be attributed to the significant rise in ASO titer and Grade 2 mitral regurgitation on echocardiography.It is theorized that symptoms are due to swelling of caudate nucleus as a result of autoimmune reaction between caudate nucleus and antineuronal antibodies which are formed against streptococcal infection [6]. Reports also suggest the correlation between acute exacerbation of neuropsychiatric symptoms and recent streptococcal infection. There is no specific time limit from the occurrence of infection to the onset of the symptoms. At the most a temporal correlation can be established.

We reported this case in order to raise awareness about this disorder which otherwise may lead to significant deterioration in scholastic performance and social adjustment amongst pre-pubertal and early adolescent children.

\section{Conclusion}

Pediatricians should investigate every presentation of a febrile illness with sore throat and myalgia in this age group as the sequelae may affect mental health of the child. Infrequent liaison and lack of knowledge can be attributed to the few case reports of this syndrome.A good cross referral between psychiatrist and pediatrician can help in reducing the morbidity associated with it.

\section{References}

[1] Swedo SE, Leonard HL, Garvey M, Pediatric autoimmune neuropsychiatric disorders associated with streptococcal infections: Clinical description of the first 50 cases. Am J Psychiatry 1998;155:264- 71

[2] Nih.gov, 'Possible Causes Of Sudden Onset OCD In Kids Broadened'. 2015.

[3] Mona Srivastava, Ganesh Shankar, M. N. Tripathi. PediatricAutoimmune Neuropsychiatric Disorders Associated With Streptococcal Infections (PANDAS): A Case Report. Journal of Clinical and Diagnostic Research 2011 November [cited: 2015 Aug 20]; 5:1298-1300.

[4] Maini, Baljeet et al. 'Pediatric Autoimmune Neuropsychiatric Disorders after Streptococcus Infection'. Indian Journal of Psychiatry 54.4 (2012): 375.

[5] Shankarnarayan A,John JK. Pediatric Autoimmune Neuropsychiatric Disorder Syndrome(PANDAS):A case report. Nat'l Med J India 2003;16 (1):22-3.

[6] Dale, R.C. 'Autoimmunity andthe Basal Ganglia: New Insights into Old Diseases'. QJM 96.3 (2003): 183-191. 